Pacific Journal of Mathematic

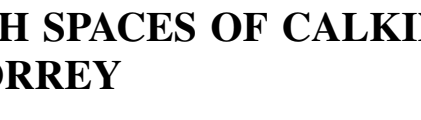




\title{
A NOTE ON THE BANACH SPACES OF CALKIN AND MORREY
}

\author{
E. H. Rothe
}

1. Introduction. Let $G$ be a bounded domain in an $n$-dimensional real Euclidean space, and for $\alpha>1$, let $L_{a}$ be the space of real-valued functions $f$ such that $f^{a}$ is summable over $G$. The class $\Re_{a}$ as defined by Calkin and Morrey $[2 ; 6 ; 7]$ is then the class of functions which together with their first "generalized derivatives" $\left[2\right.$, Def. $3.4 ; 7$, p. 4] are in $L_{\alpha}$. With a suitable norm, $\Re_{\alpha}$ becomes a Banach space [2, p. 185]. Morrey proved [7, p. 8] that in this Banach space the solid sphere $V$ of radius $K$ and with the origin as center is "weakly compact" 1 ). Using this fact together with lower semicontinuity theorems, he obtained very general existence theorems for minima of multiple integrals ${ }^{2}$ ).

The object of the present note is to point out that some of the results in this direction may be obtained by the use of general Banach space theory: the starting point is the simple remark that the Banach space $\S_{\alpha}$ is reflexive $(\$ 2)$. The weak compactness of the solid sphere $V$ is, by Alaoglu's theorem [1], a corollary to this remark [ $\$ 3]$. It now follows almost immediately that a real-valued function $I(x)$, which is "weakly" lower semicontinuous, takes a minimum in $V$ (Theorem 3.1). In $\$ 4$ some sufficient conditions for weak lower semicontinuity are given. Finally, as an example of the applicability of these considerations to calculus of variation problems, a theorem on the existence of minima of multiple integrals is given which is related to, but not identical with, the results of Morrey referred to at the end of the previous paragraph $(\$ 5)$.

2. The uniform convexity and reflexivity of the space $\Re_{\alpha^{*}}$ Let $t$ denote the point with coordinates $t_{1}, t_{2}, \cdots, t_{n}$ of the domain $G$ of $\$ 1$. Let $f(t)=f^{(0)}(t)$ be an element of $\Re_{\alpha}$, and $f^{(i)}(t)(i=1,2, \ldots, n)$ its first generalized derivative with respect to $t_{i}$. Let $\|f\|$ be defined by the equation

$$
\|f\|^{\alpha}=\int_{G} \sum_{i=0}^{n}\left|f^{(i)}(t)\right|^{\alpha} d t .
$$

${ }^{1}$ See [7] for Morrey's definition of weak compactness. The weak topology used in the present paper is defined in $\$ 3$.

${ }^{2}$ See [ 7, Chap. III], where also the relation to the results of Tonelli is discussed.

Received April 28, 1952.

Pacific J. Math. 3 (1953), 493-499 
We then have:

LEMMA 2.1. Let $\alpha>1$. With the norm defined by (2.1) the space of classes of functions of $\Re_{\alpha}$ equivalent under this norm is a Banach space ${ }^{3}$.

From now on $\Re_{a}$ will always denote the Banach space of Lemma 2.1, and it will always be supposed that $\alpha>1$.

THEOREM 2.1. The space $\mathfrak{S}_{\alpha}$ is uniformly convex ${ }^{4}$.

Proof. Let $L_{a}$ be the Banach space of classes of equivalent functions $f$ which are defined in $G$ and for which

$$
\left\{\int_{G}|f|^{\alpha} d t\right\}^{1 / \alpha}
$$

exists. $L_{a}$ is uniformly convex [3, p.403, Corollary]. Since a finite "uniformly convex" direct product of uniformly convex spaces is uniformly convex [3, p. 397398 ] it follows that the direct product of $L_{a}$ taken $(n+1)$ times by itself, that is, the space $\beta_{\alpha}^{\sim}$ of $(n+1)$-tuples $f_{0}, f_{1}, \cdots, f_{n}\left(f_{\nu} \in L_{\alpha} ; \nu=0,1, \cdots n\right)$ with the norm

$$
\left\{\int_{G} \sum_{\nu=0}^{n}\left|f_{\nu}\right|^{a} d t\right\}^{1 / \alpha}
$$

is likewise uniformly convex. This proves the theoren since $\beta_{\alpha}$ is obviously a linear subspace of $\Re_{a}^{\sim}$.

Since a uniformly convex space is reflexive $[5 ; 8]$, we have the following corollary to Theorem 2.1 .

Corollary. For $\alpha>1, \$_{\alpha}$ is reflexive.

3. The compactness of the sphere $V$. We recall first a few well-known definitions and facts. Let $E$ be an arbitrary Banach space in the strong topology, that is in the topology induced by the norm of the space. Let $K$ be a positive number, and $V$ be the solid sphere $\|x\| \leqq K$ of $E$. By $V_{K}$ we denote then the topological space whose elements are those of $V$ and whose topology is induced by the following neighborhood definition: A neighborhood of the point $x_{0}$ of $V_{K}$ is determined by a positive number $\epsilon$ and a finite number of linear continuous functionals $l_{1}(x), \ldots, l_{n}(x)$, and consists of all points $x$ of $V_{K}$ for which

${ }^{3}$ See $[2$, p. 185]. The definition of the norm given by Calkin is slightly different from the one used in the present paper. However, the proof of Lemma 2.1 is essentially unaltered.

"For the definition of the term "uniformly convex" see [3]. 


$$
\left|l_{i}(x)-l_{i}\left(x_{0}\right)\right|<\epsilon \quad(i=1,2, \ldots, n) .
$$

If $E$ is the conjugate space of another Banach space $F, E=F^{*}$, we denote by $V_{K}^{*}$ the topological space whose elements are again those of $V$, but whose topology is induced by the following neighborhood definition: A neighborhood of a point $x_{0}$ of $V_{K}^{*}$ is determined by a positive number $\epsilon$ and a finite number of elements $f_{1}, \cdots, f_{n}$ of $F$, and consists of all points $x$ of $V_{K}$ for which

$$
\left|x\left(f_{i}\right)-x_{0}\left(f_{i}\right)\right|<\epsilon \quad(i=1,2, \cdots, n) .
$$

A well-known theorem of Alaoglu [ 1 , Theorem 1.3] states that $V_{K}^{*}$ is compact. Since for a reflexive space we have $V_{K}=V_{K}^{*}$, we obtain:

LЕммA 3.1. If $E$ is reflexive then $V_{K}$ is compact.

Since a strongly closed convex subset of $V$ is also closed in the weak topology (that is, in the topology of $V_{K}$ ) we have as a consequence of Lemma 3.1 the following:

LEMMA 3.2. Let $C$ be a convex subset of $V$ which is closed in the strong topology, and $C_{K}$ the same set in the topology of $V_{K}$. Then $C_{K}$ is compact.

An easy consequence of Lemma 3.2 is:

LEMMA 3.3. Let $C$ and $C_{K}$ have the same meaning as in Lemma 3.2, and let $I(x)$ be a real-valued function which is lower semicontinuous in $C_{K}$. Then $I(x)$ reaches a minimum in some point of $C .^{5}$

The preceding lemmas, together with the corollary to Theorem 2.1 , now yield the main result of the present section:

THEOREM 3.1. Let $C$ be a bounded closed convex subset of $\Re_{a}$. Let the norms of the elements of $C$ be bounded by the positive constant $K$. Let $V$ and $V_{K}$ have the same meaning as in the first paragraph of this section, with $E$ replaced by $\Re_{a}$ and denote the set $C$ in the topology of $V_{K}$ by $C_{K}$. Then $C_{K}$ is compact, and a real-valued function $I(x)$, which is lower semicontinuous in $C_{K}$, reaches a minimum in $C$.

4. Sufficient conditions for lower semicontinuity. We prove now:

THEOREM 4.1. Let $C$ and $C_{K}$ have the same meaning as in Theorem 3.1,

${ }^{5}$ For a proof that Lemma 3.2 implies Lemma 3.3 see [9, p. 423-424]. 
and let $I(x)$ be a real-valued function defined on $C$. Then the following condition is sufficient for the lower semicontinuity of $I(x)$ on $C_{K}$ (and therefore, by Theorem 3.1, for the existence of a minimum of $I(x)$ on $C)$ : to each $x_{0} \in C$ there exists a bounded linear functional $l(x)$ such that

$$
I(x)-l\left(x_{0}\right) \geqq l\left(x-x_{0}\right)
$$

for all $x \in C$.

Proof. By definition of the lower semicontinuity we have to prove: to any given $\epsilon>0$ there exists a neighborhood $N\left(x_{0}\right)$ of $x_{0}$ in $V_{K}$ such that

$$
I(x)-I\left(x_{0}\right) \geqq-\epsilon
$$

for all $x$ in the intersection, $N\left(x_{0}\right) \cap C$. But by (4.1) the inequality (4.2) will certainly be satisfied if we choose

$$
N\left(x_{0}\right)=\left\{x|| l(x)-l\left(x_{0}\right) \mid<\epsilon, x \in V_{K}\right\}
$$

THEOREM 4.2. With the same notations as in Theorem 4.1 let $I(x)$ have first and second order Fréchet differentials $D(x, h)$ and $D^{2}(x, h, k)$ at every point $x$ of $C$. Moreover, let

$$
D^{2}(x, h, h) \geqq 0 \text { for } x \in C \text {. }
$$

Then $I(x)$ is lower semicontinuous in $C_{K}$.

Proof. From the Taylor expansion [4, Theorem 5],

$$
I\left(x_{0}+h\right)-I\left(x_{0}\right)=D\left(x_{0}, h\right)+\frac{1}{2} \int_{0}^{1} D^{2}\left(x_{0}+t h, h, h\right) d t,
$$

together with (4.3), we obtain

$$
I\left(x_{0}+h\right)-I\left(x_{0}\right) \geqq D\left(x_{0}, h\right) .
$$

This inequality shows that the assumptions of Theorem 4.1 are satisfied with

$$
l\left(x-x_{0}\right)=D\left(x_{0}, x-x_{0}\right)
$$

j. An application to a multiple integral variational problem. Let $G$ be the domain of $\S 1$ with points $t=\left(t_{1}, \cdots, t_{n}\right)$. For each $\mu=1, \cdots, m$ let $z_{\mu}(t) \epsilon$ $\Re_{\alpha}$ and let $\mathbb{I}_{\alpha}$ be the space of classes of equivalent $m$-tuples $z=\left(z_{1}(t), \cdots\right.$, $\left.z_{m}(t)\right)$ with the norm 


$$
\|z\|=\left[\int_{G} \sum_{\mu=1}^{m}\left\{|z|^{\alpha}+\sum_{\nu=1}^{n}\left|\frac{\partial z_{\mu}}{\partial t_{\nu}}\right|^{\alpha}\right\} d t\right]^{1 / \alpha} .
$$

LEMm A 5.1. Theorems 3.1 and 4.1 still hold if $\Re_{\alpha}$ is replaced by $\Pi_{\alpha}$.

This lemma is obvious from the proofs of the theorems in question.

THeOREM 5.1. Let

$$
f(t, z, p)=f\left(t_{1}, \cdots, t_{n}, z_{1}, \cdots, z_{m}, p_{11}, \cdots, p_{m n}\right)
$$

be a real-valued function of the indicated variables with the following properties:

(1) $f$ is defined for $t=\left(t_{1}, \cdots, t_{n}\right) \in G$ and for all values of the real varibles $z_{\mu}, p_{\mu \nu}(\mu=1, \cdots, m ; \nu=1, \cdots, n)$, and for the same domain of the varibles $\partial f / \partial t_{\nu}, \partial f / \partial z_{\mu}$, and $\partial f / \partial p_{\mu \nu}$ are supposed to exist;

(2) if $z_{\mu}(t) \in \Re_{\alpha}$ then the functions of $t$ obtained by replacing $z$ by $z_{\mu}(t)$ and $p_{\mu \nu}$ by $\partial z_{\mu} / \partial t_{\nu}$ in $f, \partial f / \partial z_{\mu}$, and $\partial f / \partial p_{\mu \nu}$ are in $L_{\beta}$, where $\beta$ is defined by

$$
1 / \beta+1 / \alpha=1
$$

(3) $e\left(t, z, z^{0}, p, p^{0}\right) \geqq 0$,

where by. definition

$$
\begin{aligned}
& e\left(t, z, z^{0}, p, p^{0}\right)=f(t, z, p)-f\left(t, z^{0}, p^{0}\right) \\
& \quad-\sum_{\mu=1}^{m}\left\{f_{z_{\mu}}\left(t, z^{0}, p^{0}\right)\left(z_{\mu}-z_{\mu}^{0}\right)+\sum_{\nu=1}^{n} f_{p_{\mu \nu}}\left(t, z^{0}, p^{0}\right)\left(p_{\mu \nu}-p_{\mu \nu}^{0}\right)\right\} .
\end{aligned}
$$

Under these assumptions, if

$$
I(z)=\int_{G} f\left[t_{1}, \cdots, t_{n}, z_{1}(t), \cdots, z_{m}(t), \frac{\partial z_{1}}{\partial t_{1}}, \cdots, \frac{\partial z_{m}}{\partial t_{n}}\right] d t,
$$

then there exists a

$$
z^{(1)}=z^{(1)}(t)=\left[z_{1}^{(1)}(t), \cdots, z_{m}^{(1)}(t)\right]
$$


in the sphere

$$
\|z\| \leqq K
$$

such that

$$
I(z) \geqq I\left(z^{(1)}\right)
$$

for all $z$ in the sphere (5.2).

Proof. By Lemma 5.1 and Theorem 3.1, it will be sufficient to prove that $I(z)$ is lower semicontinuous at each point $z^{0}$ of the sphere (5.2). To such $z^{0}$ we define the linear functional $l(\zeta)$ of

$$
\zeta=\left[\zeta_{1}(t), \cdots, \zeta_{m}(t)\right]
$$

by setting

$$
l(\zeta)=\int_{G} \sum_{\mu=1}^{m}\left\{\left(f_{z_{\mu}}\right)_{0} \zeta_{\mu}+\sum_{\nu=1}^{n}\left(f_{p_{\mu \nu}}\right)_{0} \frac{\partial \zeta_{\mu}}{\partial t_{\nu}}\right\} d t
$$

where the symbol ()$_{0}$ indicates that the arguments are

$$
t_{1}, \cdots, t_{n}, z_{1}^{0}(t), \cdots, z_{m}^{0}(t), \partial z_{1}^{0} / \partial t_{1}, \cdots, \partial z_{m}^{0} / \partial t_{n},
$$

and where

$$
\zeta=\left[\zeta_{1}(t), \cdots, \zeta_{m}(t)\right] \in \Pi_{a}
$$

The assumption ( 2 ) assures us that the linear functional $l(\zeta)$ is bounded. From the definition of $l(\zeta)$ and the assumption (3) we obtain

$$
I(z)-I\left(z^{0}\right)=l\left(z-z^{0}\right)+e \geqq l\left(z-z^{0}\right) .
$$

Thus the assumption (4.1) of Theorem 4.1 is satisfied, and the theorem to be proved follows from Theorem 4.1 in conjunction with Lemma 5.1.

\section{References}

1. L. Alaoglu, Weak topologies of normed linear spaces, Ann. of Math. 41 (1940), 252-267.

2. J. W. Calkin, Functions of several variables and absolute continuity 1 , Duke Math. J. 6 (1940), 170-186.

3. J. A. Clarkson, Uniformly convex spaces, Trans. Amer. Math. Soc. 40 (1936), 396414. 
4. L. M. Graves, Riemann integration and Taylor's theorem in general analysis, Trans. Amer. Math. Soc. 29 (1927), 163-177.

5. Sh. Kakutani, Weak topology and regularity of Banach spaces, Proc. Imp. Acad. Tokyo, 15 (1939), 169-173.

6. C. B. Morrey, Jr., Functions of several variables and absolute continuity, Duke Math. J. 6 (1940), 187-215.

7. - Multiple integral problems in the calculus of variations and related topics, University of California Publications in Mathematics, New Series, Vol. 1 No. 1 (1943), 1-130.

8. B. J. Pettis, A proof that every uniformly convex space is reflexive, Duke Math. J. 5 (1939), 249-253.

9. E. H. Rothe, Gradient mappings and extrema in Banach spaces, Duke Math. J. 15 (1948), 421-431.

\section{UNIVERSITY OF MichIGAN}





\section{PACIFIC JOURNAL OF MATHEMATICS}

\section{EDITORS}

\section{R. U. FOEINSON}

University of California

Berkeley 4, California

E. HewitT

University of Washington

Seattle 5 , Washington
P. P. DILWOR TH

California Institute of Technology

Pasadena 4, California

E. F. BECKENBACH

University of California

Los Angeles 24, California

\section{ASSOCIATE EDITORS}

$\begin{array}{llll}\text { H. BUSEMANN } & \text { P. R. HALMOS } & \text { BØRGE JESSEN } & \text { J. J. STOKER } \\ \text { HERBERT FFDERER } & \text { IIEINZ HOPF } & \text { PAUL LÉVY } & \text { E. G. STRAUS } \\ \text { MARSHALL, IIALI } & \text { R. D. JAMES } & \text { GEORGE PÓLYA } & \text { KÖSAKU YOSIDA }\end{array}$

\section{SPONSORS}

UNIVERSITY OF BRITISH COLUMBIA

CALIFORNIA INSTITUTE OF TECHNOLOGY.

UNIVERSITY OF CAIJIFORNIA, BERKELEY

UNIVERSITY OF CAIJIFORNIA, DAVIS

UNIVERSITY OF CALIFORNIA, LOS ANGELES

UNIVERSITY OF CALIFORNI A, SANTA BARBARA

UNIVERSITY OF NEVADA

OREGON STATE COLLEGE

UNIVERSITY OF OREGON

\author{
UNIVERSITY OF SOU TIERN CALIFORNIA \\ STANFORD RESEARCH INSTITUTE \\ STANFORD UNIVERSITY \\ WASHINGTON STATE COLLEGE \\ UNIVERSITY OF WASHINGTON \\ AMERICAN MATHEMATICAL SOCIETY \\ NATIONAL BUREAU OF STANDARDS, \\ INSTITUTE FOR NUMERICAL ANALYSIS
}

Mathematical papers intended for publication in the Pacific Journal of Mathematics should be typewritten (double spaced), and the author should keep a complete copy. Manuscripts may be sent to any of the editors except Robinson, whose term expires with the completion of the present volume; they might also be sent to M.M. Schiffer, Stanford University, Stanford, California, who is succeeding Robinson. All other communications to the editors should be addressed to the managing editor, E. F. Beckenbach, at the address given above.

Authors are entitled to receive 100 free reprints of their published papers and may obtain additional copies at cost.

The Pacific Journal of Mathematics is published quarterly, in March, June, September, and December. The price per volume (4 numbers) is $\$ 8.00$; single issues, $\$ 2.50$. Special price to individual faculty members of supporting institutions and to individual members of the American Mathematical Society: $\$ 4.00$ per volume; single issues, $\$ 1.25$.

Subscriptions, orders for back numbers, and changes of address should be sent to the publishers, University of California Press, Berkeley 4, California.

Printed at Ann Arbor, Michigan. Entered as second class matter at the Post Office, Berkeley, California.

\section{UNIVERSITY OF CALIFORNIA PRESS • BERKELEY AND LOS ANGELES}




\section{Pacific Journal of Mathematics}

\section{Vol. 3, No. 2 \\ April, 1953}

William George Bade, An operational calculus for operators with spectrum

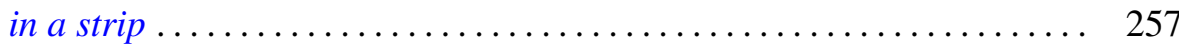

E. F. Beckenbach and Lloyd Kenneth Jackson, Subfunctions of several variables ..................................... 291

David Blackwell, Extension of a renewal theorem ................. 315

L. Carlitz, Some theorems on the Schur derivative ................ 321

Paul Arnold Clement, Generalized convexity and surfaces of negative curvature..................................... 333

Merrill M. Flood, On the Hitchcock distribution problem ............... 369

Watson Bryan Fulks, On the unique determination of solutions of the heat

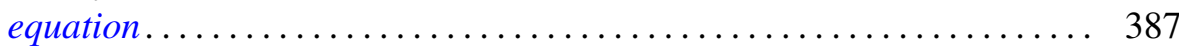

John W. Green, Length and area of a convex curve under affine transformation .................................... 393

William Gustin, An isoperimetric minimax .................. 403

Arthur Eugene Livingston, Some Hausdorff means which exhibit the Gibbs'

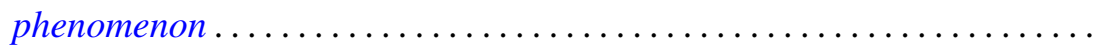

Charles Loewner, On generation of solutions of the biharmonic equation in the plane by conformal mappings ..................... 417

Gábor Szegő, Remark on the preceding paper of Charles Loewner ....... 437

Imanuel Marx and G. Piranian, Lipschitz functions of continuous

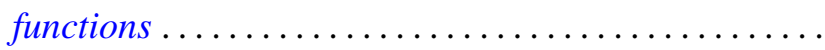

Ting-Kwan Pan, The spherical curvature of a hypersurface in Euclidean space ..

Ruth Lind Potter, On self-adjoint differential equations of second order ...

E. H. Rothe, A note on the Banach spaces of Calkin and Morrey...

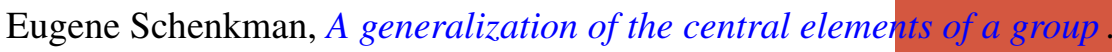

A. Seidenberg, A note on the dimension theory of rings .. . . 